\title{
Economic advantage of 'self-made' antibiotic-loaded spacer compared to prefabricated antibiotic-loaded spacer and spacer molds in two-staged revision arthroplasty
}

\author{
K. Moerenhout, S. Steinmetz, M. Vautrin, S. Picarra, G. Udin, O. Borens \\ From the Service of Orthopedics and Traumatology, Lausanne University Hospital and University of Lausanne, \\ Lausanne, Switzerland
}

Infection after total hip or total knee arthroplasty is a serious complication implying great costs for the health care system. Amongst the different treatment options, the two-step exchange using a spacer in the interval is a valid option. We evaluate the economic impact of our self-made antibiotic-loaded hip and knee cement spacers compared with prefabricated spacers and spacer molds.

Costs to prepare self-made cement spacers are detailed for each spacer type. We also assess the intraoperative time spent for fabricating our self-made hip and knee spacers.

The price of these self-made knee spacer is $514 \mathrm{CHF}$ (450 EUR / 505 USD) if non-articulated and 535 CHF (470 EUR / 525 USD) if articulated ; the price for the self-made hip spacer is 749 CHF (760 EUR / 735 USD). Our average preparation time is 14 minutes for our self-made knee spacers and $\mathbf{1 6}$ minutes for our self-made hip spacers. While the senior surgeon is fabricating the self-spacers, another surgeon of the team continues intensive irrigation and debridement. Thus, no time is lost waiting for the self-spacer to be fabricated.

In our hands, self-made hip and knee spacers are at least $40-50 \%$ cheaper than prefabricated spacers and spacer-molds. This is a serious economic advantage in this already expensive surgery. When done in teamwork, self-spacer fabrication does not increase the surgery time. The economic advantage is added to the main and most important advantage of selfmade spacers, which remains the possibility of patient adapted anatomical reconstruction of the joint.
Keywords : economic analysis ; spacer ; prosthetic joint infection; two-stage exchange.

\section{INTRODUCTION}

Infections after total hip (THA) or total knee arthroplasty (TKA) have relative incidences of respectively 2 and $2.4 \%$ (1) and this incidences can be two to three time higher after revisions of THA or TKA (2). As the number of hip and knee primary and revision prosthesis is in constant rise (3), the number of prosthetic joint infections (PJI) rises as well. PJI

Kevin Moerenhout ${ }^{1}$,

Sylvain Steinmetz',

Matthias Vautrin'1,

Sandra Cristina Picarra ${ }^{2}$,

Gilles Udin',

- Olivier Borens ${ }^{1}$.

${ }^{\prime}$ Service of Orthopedics and Traumatology, Lausanne University Hospital and University of Lausanne, Lausanne, Switzerland.

${ }^{2}$ Department of Interdisciplinary Care - Operating Room Nurse, specialized in Traumatology. Lausanne University Hospital and University of Lausanne, Lausanne, Switzerland. Correspondence : Sylvain Steinmetz, Rue du bugnon, 46, 1011 Lausanne. Fax : 00(41) 213142876.

Email : sylvain.steinmetz@chuv.ch

- 2021, Acta Orthopædica Belgica. 
is a serious complication with high morbidity and mortality, implying great costs for the health care system $(1,4)$. In order to keep those health care costs as low as possible, the surgeon must be implied in the choice of the utilized materials and techniques.

Different treatment options for PJI exist, going from conservative treatment with suppressive antibiotic therapy or iatrogenic fistulisation, to debridement and retention surgery and finally to one or two step prosthetic joint exchange (5). In contrary to one step exchange with a single hospitalization, the choice of a two-step exchange involves two hospitalizations with antibiotic therapy in the interval, implying greater costs.

The two-step exchange, which was first described in 1983 by Insall et al. (6), has demonstrated success rates of above $90 \%$ (7). Garvin and Hanssen found that two-stage procedures without antibiotic-loaded cement had a better success rate $(82 \%$ of 158 hip joints) than one-stage exchange arthroplasties (58\% of 60 hip joints), although systemic antibiotics were used for both procedures (8). With the addition of antibiotics, the rates of successful eradication of the infection increased to $91 \%$ (385 of 423 hip joints) for the two-stage technique and 82\% (976 of 1189 hip joints) for the one-stage revision (8). For many authors this two-step exchange with an antibiotic loaded cement spacer remains the gold standard in chronic infections treatment $(9,10)$.

To fill the death space after implant removal and to elude local antibiotics, prefabricated spacers or spacer molds are available, with the advantage of being easy to use. They can be made entirely of polymethylmethacrylate cement, or it may be a cement-coated metal core or a sterile prosthesis partially coated with antibiotic-impregnated cement. Negative aspects are the limited available sizes and versatility, as well as their price. Another option is the intra-operative fabrication of self-made spacer. Favorable results have been reported with each of these types of spacers.

Self-made antibiotic loaded spacers are believed to be significantly cheaper than the com-mercially available spacers (11). However, the time spent to fabricate them and the eventual increase of surgical duration has not been taken into account in different available studies (12).
The purpose of this study was to (1) determine the real economic impact of our self-made hip and knee spacers by comparing their price with prefabricated spacers and spacer molds and (2)evaluate if the surgical time is increased when self-made spacers are used.

We hypothesize that our self-made cement spacer is cheaper than other prefabricated spacers and that the fabrication of those self-made spacers does not increase surgical time.

\section{METHODS}

Intra-operative time to fabricate our self-made spacers was analyzed. Mean confection time for a hip spacer (20 patients) and knee spacer (20 patients with articulated spacer and 20 patients with nonarticulated spacer) was measured.

At the same time, the price for the different parts needed for the fabrication of a hip respectively knee spacer was evaluated.

While the senior surgeon is fabricating the spacers, another surgeon of the team continues intensive pulsed irrigation with at least 9 liters of $\mathrm{NaCl}$ and exhaustive debridement. Synovial fluid cultures and multiple tissue cultures were taken and sent for bacteriologic analysis.

Our self-made spacers, both hip and knee, were made with $2 \mathrm{~g}$ of Vancomycin and $1.2 \mathrm{~g}$ of Tobramycin per 40 cc of Palacos $R+G \AA$ cement containing already $0.5 \mathrm{~g}$ of Gentamycine (Heraeus, Wehrheim, Germany).

After removal of the infected hip prosthesis, the acetabulum is debrided and reamed $2 \mathrm{~mm}$ above the size of the extracted acetabular cup. The femoral canal is reamed until a diameter of $14 \mathrm{~mm}$. Extensive and meticulous debridement is performed. In the meanwhile, the hip spacer is fabricated. A threaded $\mathrm{K}$-wire of $3.2 \mathrm{~mm}$ guidewire and a narrow 4.5 fracture fixation plate are used to reinforce the cement in hip spacers, thus diminishing the risk of a fracture of a pure PMMA spacer (figure 1). The shaft is molded with a Thin-Flex venous cannula (Edwards, Newbury, UK), while the head is molded with the interior surface of an acetabular reamer of the desired size (13). The size of the reamer is normally 2 sizes bigger than the size of the last used 

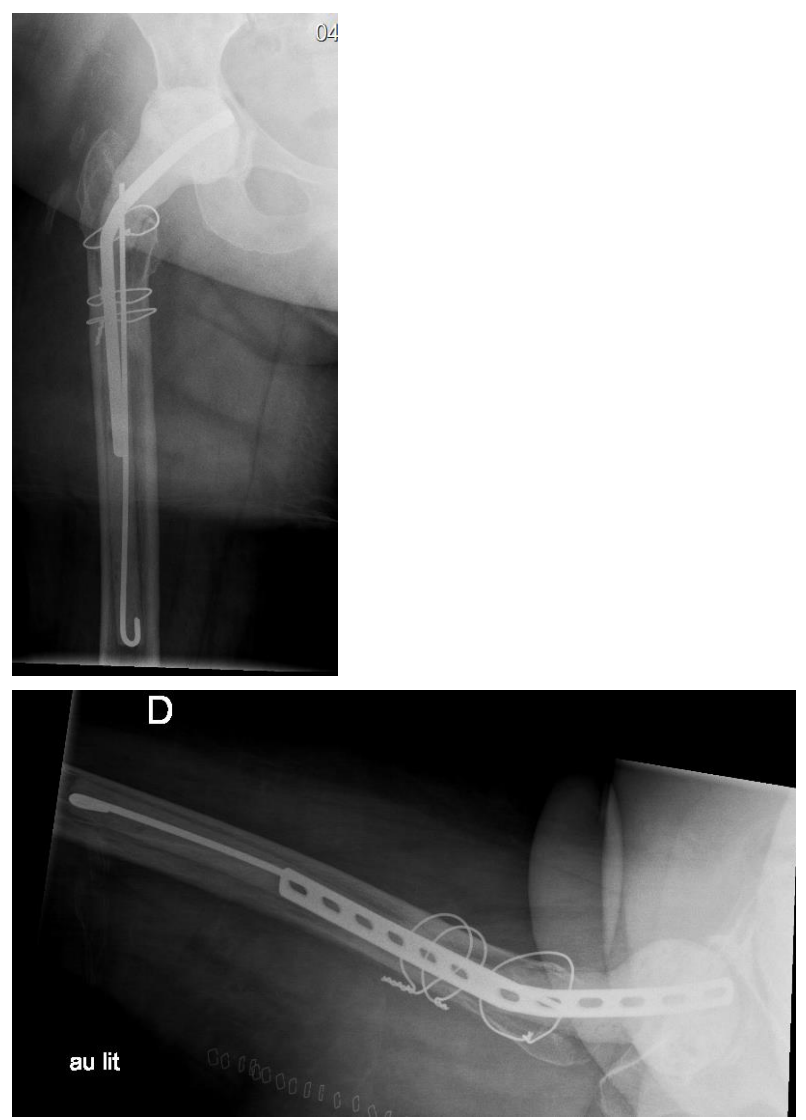

Figure 1. - Postoperative antero-posterior and axial X-ray of a right hip showing the articulated hip spacer, armed with a $\mathrm{K}$-wire and narrow 4.5 LCP plate.

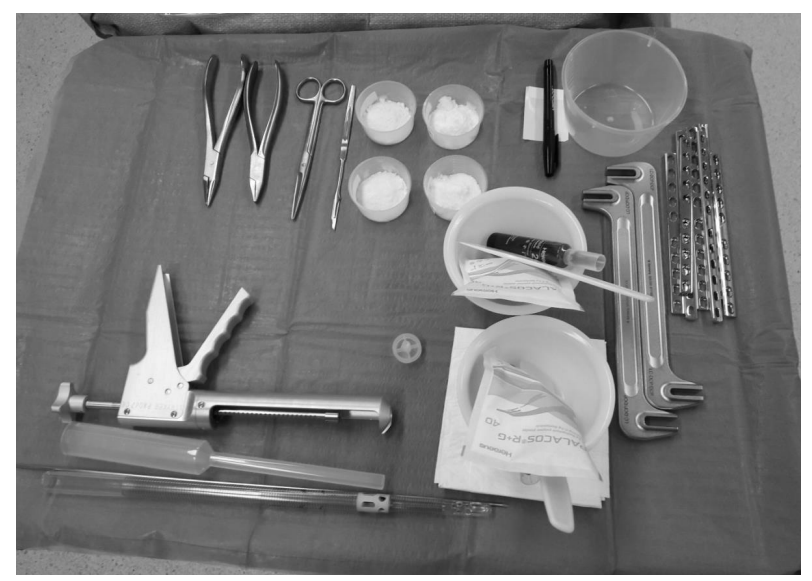

Figure 2. - Table set-up for confection of a self-made hip spacer. Four little buckets contain the antibiotics.

reamer for the cleaning of the acetabular cavity. A typical setup for confection of a hip spacer is shown in (figure 2), which represents the supplementary material needed.

Knee spacers are usually articulated but can be non-articulated in case of large bone destruction or an incompetent or fragile extensor apparatus. The main advantage of a well-molded, well-fitted articulated spacer restoring soft-tissue tension and allowing for greater degree of joint motion has been reported. It has a better outcome than the use of a non-articulated spacer, which may limit joint movement. However, these spacers can migrate, dislocate, break and cause bone loss (14).

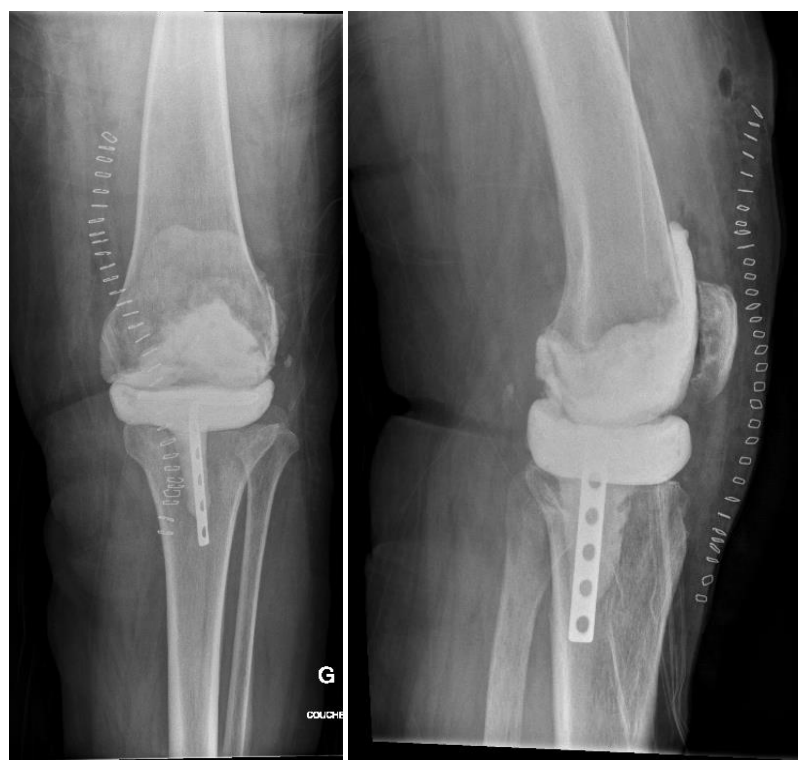

Figure 3. - Postoperative antero-posterior and lateral X-ray of a left knee showing the articulated knee spacer, armed with a one-third tubular plate on the tibial part.

In articulated knee spacers, a one-third tubular plate is bended and used to arm the tibial part of the knee spacer, while the femoral part is molded directly over the condyles of the remaining distal femur (figure 3). In non-articulated knee spacers, two 3-mm threaded wires are used to arm the cement in order to have more strength and allow a complete extraction of the cement during secondary prosthetic reimplantation (figure 4).

Prices for each table set-up was individually taken for each spacer type. Those costs were compared to prefabricated spacers and spacer molds, based on 

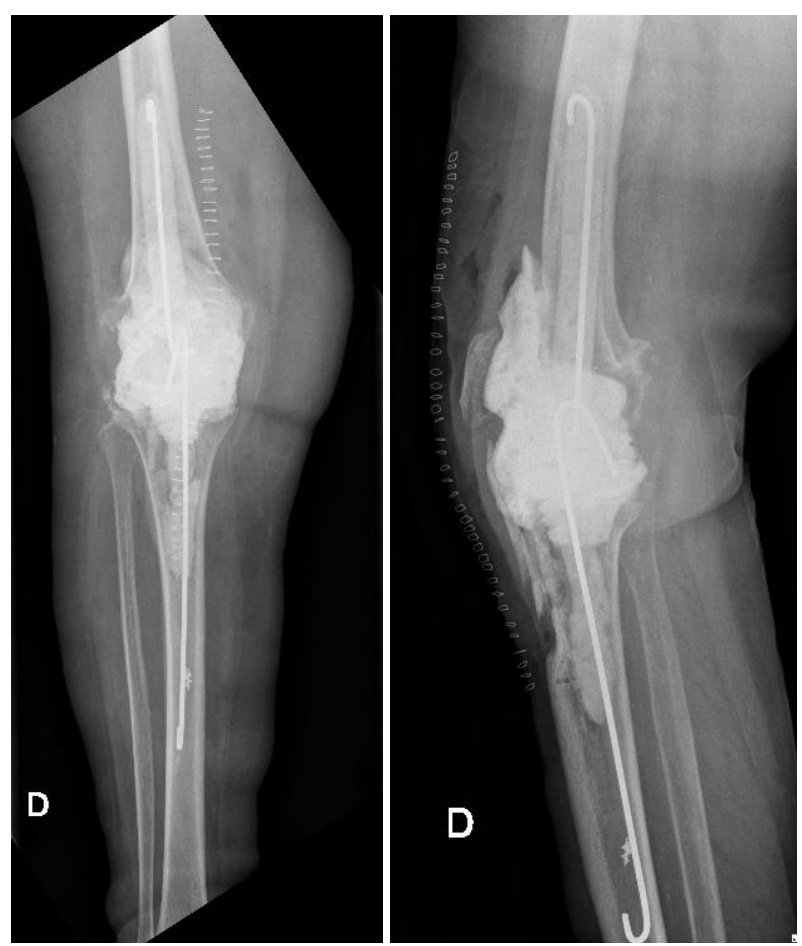

Figure 4. - Postoperative antero-posterior and lateral X-ray of a right knee with a non-articulated knee spacer. Two K-wires are used to arm the cement.

products catalog prices. The negotiated rates may vary from one institution to another, which may affect price variability between groups.

\section{RESULTS}

Our average preparation time was 14 minutes for articulated and non-articulated knee spacers (12-16 minutes) and 16 minutes (14-18 minutes) for hip spacers. While the senior surgeon is molding the spacer, meticulous debridement is continued by another surgeon of the team. After this throughout debridement, the InterPulse irrigation system (Stryker) is used for irrigation. This device has an outflow of $750 \mathrm{ml} \mathrm{min}$. Nine liters of $\mathrm{NaCl}$ are used in every case, which means a minimum of 12 minutes for irrigation alone is needed. When adding the extensive debridement to this 12 minutes irrigation, the spacer was in all 60 patients ready for use. Thus, no time is lost waiting while the spacer is being fabricated.
Table I. - Supplementary material needed for confection of the antibiotic loaded spacers

\begin{tabular}{|l|l|c|}
\hline Type of spacer & $\begin{array}{l}\text { Required instruments } \\
\text { (including sterilization) }\end{array}$ & $\begin{array}{c}\text { Price } \\
\text { (in CHF) }\end{array}$ \\
\hline \multirow{4}{*}{ Hip spacer } & Narrow DCP 4.5 plate & 140 \\
\cline { 2 - 3 } & Threaded wire & 25.5 \\
\cline { 2 - 3 } & Plate bower & 10 \\
\cline { 2 - 3 } & Cement syringe & 29.1 \\
\cline { 2 - 3 } & Edwards cannula & 75 \\
\cline { 2 - 3 } & Cement 40 g (x2) +6 bowls & 200.6 \\
\cline { 2 - 3 } & Vancomycine 1 g (x4) & 47.6 \\
\cline { 2 - 3 } (articulated) & Tobramycine 1,2 g (x2) & 221.5 \\
\hline \multirow{5}{*}{$\begin{array}{l}\text { Knee spacer } \\
\text { (nnon-articulated) }\end{array}$} & $1 / 3$ tubular plate (12-holes) & 65.3 \\
\cline { 2 - 3 } & Cement 40 g (x2) +6 bowls & 200.6 \\
\cline { 2 - 3 } & Vancomycine 1 g (x4) & 47.6 \\
\cline { 2 - 3 } & Tobramycine 1,2 g (x2) & 221.5 \\
\cline { 2 - 3 } & Cement 40 g (x2) + 5 bowls & 193.9 \\
\cline { 2 - 3 } & Vancomycine 1 g (x4) & 47.6 \\
\cline { 2 - 3 } & Tobramycine 1,2 g (x2) & 221.5 \\
\hline
\end{tabular}

The mean price for our self-made hip spacer is 749 CHF (760 EUR / 735 USD), the mean price for our self-made knee spacer is 514 CHF (450 EUR / 505 USD) if non-articulated and 535 CHF (470 EUR / 525 USD) if articulated (Table 1). In comparison, those prices are in average minimum $1250 \mathrm{CHF}$ (= 1097 EUR / 1226 USD) for hip spacers, respectively minimum $1050 \mathrm{CHF}$ (922 EUR / 1030 USD) for prefabricated knee spacers and knee molds spacers.

No breakage of our spacers has occurred. One hip spacer dislocation has been reported; and one non-articulated knee spacer dislocation in a noncompliant patient.

\section{DISCUSSION}

In our hands, self-made hip and knee spacers are minimum $40-50 \%$ cheaper than prefabricated spacers and spacer molds. This is a major economic advantage in this already expensive surgery (15). In a DRG system, this difference cannot be neglected. The cost is substantially different between the groups.

This economical advantage is added to the main advantage of self-made spacers, which remains the possibility of anatomical reconstruction of the 
joint. Prefabricated spacers have a limited number of sizes, which do not always respect the anatomy of the patient (16). The versatility is hence less satisfying for the surgeon and the patient with those prefabricated spacers.

When done in teamwork, spacer preparation does not increase the surgery time. While one surgeon does the extensive debridement required after PJI, the other fabricates the antibiotic loaded spacer. This implies at least one assistant for the surgery, which is the case in our daily practice for revision surgery. The time spent for the pulsatile $\mathrm{NaCl}$ lavage with the device we use is 12 minutes, without the debridement that goes with it. Our selfmade spacers are thus not responsible for a longer operation time.

The choice of articulated versus non-articulated knee spacers depends on the available bone stock and the competence of the extensor apparatus. In presence of those conditions, we prefer articulated spacers as it has shown better final range of motion in different studies $(17,18)$. There is however no difference in infection recurrence rate between those two types of spacers. The most important variables during the surgical part of a two-stage exchange may be the thorough irrigation and debridement with removal of all foreign bodies at the time of explantation, rather than the type of spacer used or amount of additional antibiotic added to the cement. Fehring et al. found that the infection eradication rates $(86 \%$ in the static group, $92 \%$ in the articulating group, $\mathrm{P}=0.06$ ) were similar in the 2 groups, and there was no statistically significant difference in Hospital for Special Surgery score and range of motion (14).

Emerson at al. found that the reinfection rates were similar $(7.6 \%$ in the static group, $9 \%$ in the articulating group, $\mathrm{P}=0.8 \%$ ), and the articulating group had a better range of motion than the static group (107.8 $8^{\circ}$ compared with $\left.93.7^{\circ}, \mathrm{P}=.01\right)$ (19). They compared 26 patients treated with a nonarticulated spacer (average duration of follow-up, 7.5 years) with twenty-two treated with an articuled spacer (average duration of follow-up, 3.8 years) and found a $9 \%$ reinfection rate in the patients with the articuled spacer compared with an $8 \%$ rate in those with a non-articuled spacer. However, the range of motion of the joints with an articuled spacer was an average of $14^{\circ}$ greater.

An advantage of self-made spacer is the availability of the implants. In prefabricated spacers or mold spacers, implants are not available in all sizes and the existing sizes are already creating a large stock to handle with.

The strength of this article is the economic analysis of self-made and patient adapted hip and knee spacers. The prices presented are those obtained by our hospital, and don't apply exactly to all hospitals. The price difference however is absolutely in advantage of the self-made spacers.

\section{CONCLUSION}

In our hands, self-made hip and knee cement spacers are cheaper than prefabricated spacers and mold spacers. This is a serious economic advantage in this already expensive surgery. When done in teamwork, spacer fabrication does not increase the surgical time. This economical advantage is added to the main and most important advantage of self-made spacers, which remains the possibility of patient adapted anatomical reconstruction of the joint. We did witness few spacer related complications.

\section{Author's contributions}

MK and BO conceived and designed the study. VM and UG analyzed the data from PS. MK, SS and BO helped with data interpretation. MK and SS wrote the initial manuscript draft which was then revised appropriately by all authors.

\section{REFERENCES}

1. Kurtz SM, Lau E, Watson H, Schmier JK, Parvizi J. Economic burden of periprosthetic joint infection in the United States. J Arthroplasty. 2012 Sep ; 27(8 Suppl) : 61-5.e1.

2. Voigt J, Mosier M, Darouichec R. Antibiotics and antiseptics for preventing infection in people receiving revision total hip and knee prostheses : a systematic review of randomized controlled trials. BMC Infect Dis. 2016 Dec $12 ; 16(1): 749$.

3. HCUPnet. http://hcupnet.ahrq.gov/ performed on $8 / 26 / 15$ for primary and revision THA and TKA for the 2006-2012 timeframes. 
4. Hebert CK, Williams RE, Levy RS, Barrack RL. Cost of treating an infected total knee replacement. Clin Orthop Relat Res. 1996 ; $331:$ 140-5.

5. Zimmerli W, Trampuz A, Ochsner PE. Prosthetic-joint infections. N Engl J Med. 2004 Oct 14 ; 351(16) : 1645-54.

6. Insall JN, Thompson FM, Brause BD. Two-stage reimplantation for the salvage of infected total knee arthroplasty. J Bone Joint Surg Am. 1983 ; 65 : 1087-98.

7. Biring GS, Kostamo T, Garbuz, et al. Two-stage revision arthroplasty of the hip for infection using an interim articulated Prostalac hip spacer : a 10- to 15-year follow-up study. J Bone Joint Surg Br 2009 ; 91 : 1,431-1,437.

8. Garvin KL, Hanssen AD. Infection after total hip arthroplasty. Past, present, and future. J Bone Joint Surg Am. 1995 ; 77 : 1576-88.

9. Sanchez-Sotelo J, Berry DJ, Hanssen AD, Cabanela ME. Midterm to long-term followup of staged reimplantation for infected hip arthroplasty. Clin Orthop Relat Res. 2009 Jan ; 467(1) : 219-24.

10. Toulson C, Walcott-Sapp S, Hur J. et al. Treatment of infected total hip arthroplasty with a 2-stage reimplantation protocol : update on "our institution's" experience from 1989 to 2003. J Arthroplasty. 2009 Oct ; 24(7) : 1051-60.

11. Nodzo SR, Boyle KK, Spiro S, Nocon AA, Miller AO, Westrich GH. Success rates, characteristics, and costs of articulating antibiotic spacers for total knee periprosthetic joint infection. Knee. 2017 Oct ; 24(5) : 1175-1181.
12. Shen H, Zhang X, Jiang Y, Wang Q, Chen Y, Wang Q, Shao J. Intraoperatively-made cement-on-cement antibiotic-loaded articulating spacer for infected total knee arthroplasty. Knee. 2010 Dec ; 17(6) : 407-11.

13. Baker RP, Borens O. A versatile hip spacer for first stage revision in prosthetic joint infection. Ann R Coll Surg Engl. 2015 Mar ; 97(2) : 163.

14. Fehring TK, Odum S, Calton TF, et al. Articulating versus static spacers in revision total knee arthroplasties. The Ranawat Award. Clin Orthop 2000 ; 380 : 9.

15. Fischbacher A, Peltier K, Borens O. Economic Analysis in a Diagnosis Related Groups System for Twostage Exchange of Prosthetic-joint Infections. $J$ Bone $J t$ Infect. 2018 Nov 24 ; 3(5) : 249-254.

16. Baker RP, Furustrand Tafin U, Borens O. Patient-adapted treatment for prosthetic hip joint infection. Hip Int. 2015 Jul-Aug ; 25(4) : 316-22. doi : 10.5301/hipint.5000277. Epub 2015 May 20.

17. Pivec R, Naziri Q, Issa K, Banerjee S, Mont MA. Systematic review comparing static and articulating spacers used for revision of infected total knee arthroplasty. J Arthroplasty. 2014 Mar ; 29(3) : 553-7.e1.

18. Ding H, Yao J, Chang W, Liu F. Comparison of the efficacy of static versus articular spacers in two-stage revision surgery for the treatment of infection following total knee arthroplasty : a meta-analysis. J Orthop Surg Res. 2017 Oct 17 ; 12(1) : 151.

19. Emerson Jr RH, Muncie M, Tarbox TR. Comparison of a static with a mobile spacer in total knee infection. Clin Orthop 2002 ; 404 : 132. 